\title{
LA DISPARITION DE LA POLITIQUE : LE RAP ENTRE ISRAËL ET LA PALESTINE, ENTRE JUIFS ET ARABES
}

Anna Zielinska

\author{
La Découverte | «Mouvements »
}

2018/4 n 96 | pages 102 à 110

ISSN 1291-6412

ISBN 9782348040870

Article disponible en ligne à l'adresse :

https://www.cairn.info/revue-mouvements-2018-4-page-102.htm

Distribution électronique Cairn.info pour La Découverte.

(C) La Découverte. Tous droits réservés pour tous pays.

La reproduction ou représentation de cet article, notamment par photocopie, n'est autorisée que dans les limites des conditions générales d'utilisation du site ou, le cas échéant, des conditions générales de la licence souscrite par votre établissement. Toute autre reproduction ou représentation, en tout ou partie, sous quelque forme et de quelque manière que ce soit, est interdite sauf accord préalable et écrit de l'éditeur, en dehors des cas prévus par la législation en vigueur en France. Il est précisé que son stockage dans une base de données est également interdit. 


\section{La disparition de la politique : le rap entre Israël et la Palestine, entre Juifs et Arabes}

\author{
Par Anna
}

ZIELINSKA*
Le rap israélien aurait pu être un instrument de revendication puissant, et sur ses marges il l'est certainement. Ce texte présente toutefois l'histoire du rap tel qu'il est fait par des artistes juifs et arabes très populaires, pour montrer leurs tentatives inabouties de s'emparer du conflit israélo-palestinien sur un fond plus général de dépolitisation de la société israélienne contemporaine.
* Membre du comité de rédaction de Mouvements.
$\mathrm{L}$ es discussions politiques en Israël sont aujourd'hui d'une difficulté presque indépassable, et tout particulièrement celles sur le conflit israélo-palestinien. La culture populaire (musique, séries) semble être une des seules sphères de dialogue réel entre des communautés. Cela pourrait être vu comme un triomphe de la culture, et il faut sans doute l'interpréter ainsi dans une certaine mesure. Toutefois, le fait que la politique soit actuellement examinée bien plus par la fiction et l'art que par de véritables actions politiques positives est un signe fort de l'échec de la politique comme projet volontariste.

L'histoire du rap juif israélien mainstream illustre de façon frappante ce processus du désinvestissement de la sphère politique, en incarnant l'évolution récente de la société israélienne. Dans ce qui suit, il s'agira de l'analyser à partir du paysage plus général du conflit israélo-palestinien vécu à travers le Hip hop. Le contexte économique et géopolitique d'Israël, ainsi que les relations avec les Palestinien'ne's - dont la violence ne fait que croître - ont rendu les Israélien·ne·s de plus en plus indifférent·e·s à la question de savoir quel est véritablement le projet politique du pays et comment celui-ci devrait évoluer. L'incertitude quant à l'avenir du pays place plus que jamais les préoccupations individuelles et les problèmes des membres de la famille et des amis au centre de la vie quotidienne en Israël. La sphère politique est définitivement associée à la corruption et aux intérêts personnels d'une élite. Selon les données recueillies en 2006, les Israélien·ne·s sont parmi celles et ceux qui ont le moins tendance à 
participer à des manifestations politiques (24,6\% l'ont fait par le passé, contre 47,6\% des Grec.que.s, 39,7\% des Français.e.s ou 34,1\% des Allemand·e·s ; 44,4\% des Israélien·ne·s ont déclaré qu'elles·ils n'étaient pas disposée.e.s à manifester dans un avenir proche) ${ }^{1}$. Cet article montrera comment le rap suit et accompagne cette dépolitisation.

Le rap est devenu populaire en Israël à la fin d'une époque, au crépuscule de l'utopie d'un État socialiste et à la dissolution des kibboutzim. Ce qui devait être une construction socialiste et horizontale est devenu un pays doté d'une armée forte, d'un ministère des Finances solide, mais où la méfiance envers l'État va croissant, et dont les citoyen'ne·s n'ont plus d'intérêt pour la politique en tant que projet positif. Avec la victoire du parti de droite Likoud en 1977, un nouveau projet étatique a été initié, celui du néolibéralisme qui prétend évacuer le besoin même de politique $^{2}$. Le processus du désinvestissement affectif de la sphère politique a été clôturé par une série de chocs : l'échec des accords d'Oslo, l'assassinat d'Itzhak Rabin en 1995, le retrait d'Israël de Gaza en 2005 et enfin la victoire subséquente du Hamas sur ces territoires, rendant impossible toute négociation. Ces événements ont conduit non seulement les personnes proches de la droite, mais de facto aussi le centre et une grande partie de la gauche vers un état d'hébétude, sinon de désespoir réel, associé à un sentiment d'impuissance dans la sphère politique ${ }^{3}$.

\section{- Hip hop et Intifada}

Jusqu'en 2000, le rap/Hip hop israélien était un genre plutôt marginal, même si plusieurs artistes intéressants et originaux avaient introduit des éléments du rap dans leur musique depuis le milieu des années 19904. Les événements de la deuxième Intifada offrent au rap et au Hip hop une opportunité unique de devenir véritablement mainstream. Un groupe d'artistes connu sous le nom "TACT "(Tel Aviv City), qui, à ses débuts, se contentait de jouer avec des éléments artificiels de la culture rap américaine, commence à exprimer des valeurs qui lui sont propres. Le personnage-clé de cet ensemble est Subliminal (Kobi Shimoni, né en 1979), principal représentant, pendant des années, du "rap sioniste", souvent en collaboration avec The Shadow (Hatzel/Yoav Eliasi, né en 1977).

Les paroles de leurs premières chansons ne font que rarement allusion aux questions de l'exclusion sociale, car ils ne sont pas issus de milieux sociaux défavorisés. Tout comme en Afrique et en Europe de l'Est, la culture du Hip hop américain a d'abord été adoptée par des milieux économiquement aisés. Leurs débuts sont marqués par des préoccupations tout autres : la montée des tensions entre Palestinien'ne·s et Israël après les accords d'Oslo et la critique internationale de la politique
1. Voir le texte de Y. Yishai $i n:$ T. Hermann (éd.), By the People, For the People, Without the People? The Emergence of (Anti) Political Sentiment in Israel and in Western Democracies, Jerusalem, The Israel Democracy Institute, 2011, https:// en.idi.org.il/media/6371/ eb1bythepeople.pdf

2. Voir A. MARON et M. Shalev (dir.), Neoliberalism as a State Project: Changing the Political Economy of Israel, Oxford, New York, Oxford University Press, 2017 ; ainsi que D. Rosenblum, "The Future of Bibi-ism ", Haaretz, 14/09/2012.

3. D. Grossman, Chroniques d'une paix différée, trad. fr. J.-L. Allouche, Paris, Le Seuil, 2003.

4. Par exemple le groupe Shabak Samech, des musiciens réellement originaux, dont le style pourrait être comparé à Rage Against the Machine.

\section{Le processus du désinvestissement affectif de la spbère politique a été clôturé par une série de chocs : l'échec des accords d'Oslo, l'assassinat d'Itzhak Rabin en 1995...}


israélienne. Le premier album de Subliminal, Haor m'Zion (La Lumière de Sion, 2000), évoque notamment la nostalgie d'un passé plus ordonné, parle des kamikazes, et de la critique d'Israël, qu'il considère comme injustifiée. Avec cet album, où il manifeste une certaine audace caractéristique des Israéliens, celle d'un garçon grossier qui parle avec son cœur, et créé un mélange unique entre l'esthétique gangsta et la franchise israélienne, il parvient à capter les sen-

Le premier succès du rap israélien n'est donc pas associé à une révolte contre la politique nationale dominante, mais plutôt à une légitimation de cette dernière. timents du public de cette époque. Les paroles des chansons de cette période diffèrent largement de ce que font les artistes américains, puisqu'elles expriment des sentiments patriotiques et se situent politiquement à droite. Le premier succès du rap israélien n'est donc pas associé à une révolte contre la politique nationale dominante, mais plutôt à une légitimation de cette dernière. "Les gens me disent toujours : "Le rap est une musique de protestation, mais un garçon d'un bon quartier n'a pas de quoi se révolter ". Mais mes chansons ne disent pas qu'il n'y a pas assez à manger ; elles disent qu'il n'y a pas assez de fierté dans mon pays ", déclare Subliminal en mai 2000.

Subliminal et The Shadow ne soutiennent en apparence pas de parti politique particulier, et semblent s'opposer aux divisions politiques traditionnelles. Dans Divide and Conquer, ils chantent: "Les politiciens de droite et de gauche divisent et gouvernent [...]; nous survivrons ensemble, nous tomberons ensemble". Les rappeurs adoptent ici une stratégie classique du populisme, suggérant une mythique union des "peuples ", indépendamment de la politique traditionnelle, au nom d'un idéal qui n'est jamais véritablement explicité, et sous l'égide d'un leader charismatique. Dès ses origines, le rap israélien adopte cette posture indifférente à l'égard de la politique classique, reflétant un processus qui s'est aujourd'hui étendu à toute la société.

\section{- Le rap des Arabes et avec les Arabes}

L'émergence du "rap sioniste " s'accompagne de celle de son homologue arabe, le Hip hop palestinien ou pro-palestinien, venu d'Israël et de Gaza. Au début des années 2000, des artistes juifs et arabes coopèrent. Subliminal et The Shadow invitent souvent Tamer Nafar (né en 1979, fondateur, avec son frère, du groupe DAM), un artiste arabe israélien, à les rejoindre sur scène. Plusieurs de ces rencontres sont filmées ${ }^{5}$, ce qui permet aujourd'hui de revoir Subliminal poser à Nafar la question : "Israël est mon pays. Es-tu avec moi ?". Ce dernier répond : "Seul le Hip hop apportera la paix".

Néanmoins, au cours des mois qui suivent la deuxième Intifada, Tamer Nafar devient de plus en plus explicite au sujet de l'occupation israélienne. L' " ami arabe " devient un "provocateur ". Après avoir chanté la misère d'une enfance à Lod, il commence à aborder des sujets d'une portée 
plus générale : l'année 1948, "Nakba ", marquant le début du déplacement souvent forcé des Palestinien·ne·s après la création de l'État d'Israël. Et tout comme le rap juif israélien fait référence à Tel Aviv ou à Petah Tikva (il en sera question plus loin), le rap arabe israélien, par la voix de Nafar, parle de Lod, une ville en déclin près de l'aéroport Ben Gourion. Les vidéos musicales

Au cours des mois qui suivent la deuxième Intifada, Tamer Nafar, l'"ami arabe », devient

\section{un "provocateur».}

qui ressemble à Gaza, région à laquelle elles font souvent allusion.

Nafar a commencé par chanter en hébreu et en anglais, mais il adopte progressivement l'arabe, pour être compris par le public arabophone. Il signale, dans le documentaire de Halachmi, que lorsqu'il parlait en hébreu devant un public juif, qu'il disait être arabe et commençait à évoquer les difficultés de sa communauté, il avait l'impression qu'ils le trouvaient "mignon" (cute). Le changement de ton est notamment marqué par la chanson Me'en Erhabe (Qui est le terroriste?), jouée par DAM depuis 2000 et sortie en ligne en 2001. Un succès auprès du public arabophone, et l'une des raisons de la fin de l'amitié entre les artistes TACT et Nafar, en particulier à cause de la phrase : "La démocratie ? Vous ressemblez plutôt à des nazis! ». Le rap afro-américain, qui a d'abord été pour le leadeur de DAM une esthétique (découverte par le biais des musiciens juifs), commence à devenir une source d'inspiration pour parler de la lutte palestinienne pour l'indépendance. Pour Subliminal en revanche, le contexte afro-américain du rap a peu d'impact sur sa compréhension de la situation politique en Israël ${ }^{6}$.

Même si les artistes TACT disent à ce moment-là qu'ils n'ont " rien contre Tamer [Nafar] ", ils expriment un désaccord de principe sur l'utilisation de la musique pour véhiculer des messages politiques: "Nous venons ici pour débrancher ", "pour nous amuser ", selon Subliminal. Le fait que, dans ses chansons, Nafar compare la situation des Palestiniens à celle des femmes violées, ou qu'il procède à des analogies entre les Juifs et les Nazis, met Subliminal et The Shadow mal à l'aise face à l'idée de continuer à l'inviter à se produire dans le club qu'ils ont eux-mêmes fondé.

La position de Nafar se cristallise, à ce moment-là, en une opposition de plus en plus explicite à la politique israélienne à l'égard des Arabes, et cela dans le contexte de l'omniprésence médiatique des combattants palestiniens lançant des pierres contre les soldats de l'IDF. Il dit notamment : "Les pierres que je lance sont des rimes ", déclarant protéger "les Arabes à travers le micro ". Dans une interview, il dit qu'aussi bien luimême que les Palestiniens sont " construits à partir de la colère " (built on anger). En 2005, il admet toutefois que l'analogie entre le nazisme et Israël est non seulement fausse, mais aussi contre-productive, car elle n'a pas réussi à véhiculer le message qu'il cherchait à transmettre?

DAM trouve un appui fort dans le milieu artistique juif israélien. Dès 2000, Aviv Geffen, célèbre chanteur (également connu pour ses prises
6. Voir U. Dorchin, "Flowing beyond sectarian ethno-politics: ethnography of Jewish and Arab rap in Israel ", Ethnopolitics, 2017, vol. $16, \mathrm{n}^{\circ} 2$, p. $145-160$ $\&$ "In quest of truth: the meaning of message making in Israeli rap ", Popular Music, 2015. vol. $34, n^{\circ} 3$, p. 452-470.

7. D. LYNSKEY, "The Great Divide ", The Guardian, 11 mars 2005, http://www. theguardian.com/ music/2005/mar/11/ popandrock. 
de position de gauche), soutient le groupe, aussi bien dans ses apparitions publiques que dans son travail artistique. Ils enregistrent ensemble le morceau Innocent Criminals, chanté en hébreu pour être compris par le public juif israélien. "On dit que les Arabes sont primitifs et agressifs. Nous ne le sommes pas ", chante Nafar au nom de la communauté qu'il déclare représenter; "Avant de nous juger, marchez dans nos chaussures ". Geffen conclut la chanson avec un autre " nous ", celui des Israéliens juifs :

Nous avons conquis un peuple dont nous avons maintenant peur

Il vaut mieux avoir peur que d'être coupable

Nos cours sont parfois confus

D'un côté, nous avons des terroristes, et de l'autre - des colons.

Les dirigeants corrompus ne font que crier des slogans [...]

Une autre chanson " mixte" de cette époque est Summit Meeting, coenregistrée par Nafar avec le rappeur Sagol 59 (Chen Rotem) et Shaanan Streett de Hadag Nahash.

Ces " conversations " à travers des chansons et des interviews ont fait que Nafar et les artistes TACT cessent d'avoir de vrais échanges. Les hos-

\section{"Nous avons conquis un peuple dont nous avons maintenant peur. Il vaut mieux avoir peur que d'être coupable."} tilités sont déclarées non seulement par les chanteurs, mais aussi, sinon surtout, par leurs publics. Il n'est pas rare d'entendre le cri de "Mort aux Arabes!" pendant les concerts de Subliminal, à quoi ce dernier répond : "Ne dites pas ça. C'est " la vie pour les Juifs ", pas la mort pour les Arabes! "; "Celui qui crie "Mort aux Arabes ", va te faire foutre! " (voir le film de 2003).

Avec le temps, Subliminal a évacué de ses chansons toute dimension politique. Ses morceaux récents sont purement divertissants et reflètent divers héritages musicaux, africains entre autres. L'âge d'or du rap " de droite "s'est donc terminé dans les années qui ont précédé 2010, et ce genre musical lui-même a connu une phase de mort imminente. L'esthétique du rap a été à ce moment reprise par un artiste jouant sur le second degré, Lukach, qui parodiait à la fois Subliminal et la culture pop

8. Cet article s'intéresse à la politique israélienne surtout du point de vue du conflit israélopalestinien et des choix concernant la forme de l'État. Certaines revendications politiques liées à des questions sociales sont présentes dans le rap fait par les rappeur éthiopiens (cf. Café Shahor Hazak ou KGC) ou russes. américaine en général. À la fin de la première décennie du $21^{\mathrm{e}}$ siècle, ce dernier a écouté un enregistrement qui lui a été remis par un très jeune rappeur de Petah Tikva, Nechi Nech, ce qui a conduit à la découverte de celui-ci par le grand public. Avec Tuna, Nechi Nech incarne désormais le mainstream du rap israélien, et de la musique indépendante israélienne tout court. Leur travail est incomparablement plus professionnel que celui de leurs prédécesseurs, et leur positionnement à l'égard de la politique est également très différent : ils l'ignorent, aujourd'hui encore ${ }^{8}$.

\section{- La nostalgie de Petah Tikva}

Parmi les artistes de rap qui viennent d'être cités, beaucoup viennent de Petah Tikva, une ville proche de Tel-Aviv, qui est devenue le point de 
référence de plusieurs morceaux, un lieu évoqué avec nostalgie. Une certaine mélancolie semble être devenue la toile de fond, surprenante, du rap israélien de la fin de la deuxième décennie du XXI'siècle. Elle évoque un passé réellement vécu autant qu'un passé collectif mythique.

Nechi Nech (jeu de mots avec un mot amharique/éthiopien "blanc ", näch ; Ravid Plotnik, né en 1988), joue avec plusieurs héritages culturels, réels ou imaginaires, sans connotation politique. Son désir d'authenticité et de sincérité est devenu sa marque de fabrique, et les thèmes évoqués sont notamment l'insécurité personnelle et la construction de soi, l'amitié, la recherche de l'amour réel, les liens privilégiés formés pendant l'enfance et l'adolescence. Il emploie des éléments qui viennent soit de l'Ouest (comme dans la chanson Yiddishe Rastaman, 2013, mélangeant des éléments de reggae et de klezmer, c'est-à-dire de la culture juive d'Europe de l'Est), soit de l'Orient (Rap King of the Middle East, 2015, fait allusion musicalement et visuellement à l'esthétique arabe). Les chansons qui proposent une critique sociale ou politique d'Israël ont une portée universelle, s'appliquant aux évolutions de la société occidentale au sens large. Iron age (2014), par exemple, critique un rêve israélien devenu un cauchemar, où la diversité et l'espoir ont été transformés en une recherche désespérée de l'homogénéité et une obsession de la sécurité. L'album Welcome To Petach Tikva (2015), comprend notamment un morceau qui imite le blues classique, en insistant sur le fait que le "gouvernement est un crime organisé ", et sur le "système " qui ne permet pas de prendre des décisions personnelles essentielles - sur un ton, toutefois, parodique. Toutes les chansons sont en hébreu, toutes contribuent de manière légèrement teintée d'autodérision à créer une histoire commune de la génération née dans les années 1980, qui partage la même formation au sein des mêmes banlieues sinistres.

Cette cristallisation d'une expérience générationnelle partagée façonnée par des objets et des phénomènes banals et ordinaires plutôt que par de grands mouvements historiques a été confirmée en 2016 par une chanson d'un autre artiste de Petah Tikva, Tuna (Itay Zvulun, né en 1984), Gam zeh yavor (Cela aussi va passer). La phrase - classique en hébreu, dont les origines sont persanes-qui fait le titre de la chanson est habituellement

\section{Les chansons qui proposent une critique sociale ou politique d'Israël ont une portée universelle, s'appliquant aux évolutions de la société occidentale au sens large.} employée pour parler des luttes existentielles et de l'absurdité de l'attachement humain aux contingences mondaines. Ici, elle est répétée à un ami qui vit une rupture amoureuse, pour lui dire que sa douleur passera, tout comme tant d'autres éléments de notre passé : Samantha Fox, le Walkman, un vieux club culte de Tel Aviv ou d'autres encore, tous appartenant à l'univers de la fin des années 1980 et 1990 en Israël et dans le monde, et créant un sentiment de communauté générationnelle. Une autre chanson célèbre du premier album 
de Tuna, écrite pour son $30^{\mathrm{e}}$ anniversaire (Rock Shloshim), s'arrête elle aussi sur les interrogations personnelles et existentielles d'une génération dont le succès ne semble mesurable que par le degré de participation au monde de la high-tech et à

"Vous samplez sur la musique arabe ", dit Nafar en direction des rappeurs commerciaux israéliens. la "start-up Nation " (slogan publicitaire d'Israël) : "Qu'es-tu prêt à abandonner? Combien reste-t-il à accomplir?" Le second album de l'artiste, Tuna Park (2017), évoque souvent un sentiment d'aliénation

résultant de sa troublante popularité, acquise après le succès de son premier album. Cela amène son travail à un second niveau de dépolitisation, en entrant dans un univers d'autoréférences.

Le seul morceau de Tuna qui fait allusion au monde arabe au sens large est Seret Aravi (Film arabe, 2016), un hommage humoristique aux films d'action kitsch égyptiens des années 1970 et 1980. La vidéo de la chanson imite l'esthétique de ce cinéma, et les paroles renforcent cet effet en ajoutant des terminaisons à consonance arabe à des mots hébreux et anglais. Il ne s'agit pas ici de se moquer de ces films de série B (voire C ou Z); au contraire, le morceau ne fait que les inclure dans le patrimoine culturel partagé des décennies précédentes. Néanmoins, sa tendance à effacer toute référence au contexte politique qui mine les relations dans le monde arabe, à savoir la relation avec les Palestiniens, a provoqué une réaction de la part de Tamer Nafar et de DAM.

Dans un morceau qui reprend la même ligne mélodique que Seret Aravi, Nafar annonce, en un mélange d'hébreu, d'anglais, d'arabe et d'allemand/yiddish, la " parodie d'une parodie ". Il entend critiquer précisément la chanson de Tuna, mais en profite pour dénoncer aussi ce qu'il perçoit comme une forme d'appropriation culturelle: "Vous samplez sur la musique arabe ", dit Nafar en direction des rappeurs commerciaux israéliens (les accusations analogues d'appropriation culturelle apparaissent dans d'autres genres musicaux, dans la pop notamment). En jouant avec l'allemand ou le yiddish, on devine qu'il suggère, cette fois de façon non-explicite, l'existence de liens entre l'Allemagne nazie et l'Israël contemporain. Il continue en critiquant la culture juive israélienne pour son machisme, pour terminer avec l'accusation plus fondamentale d'instrumentalisation du rap : "Vous faites du Hip hop pour des boîtes de striptease [allusion à un scandale lié à The Shadow], moi je le fais pour la bande de Gaza ".

9. Voir T. Hermann,

C. Cohen, E. Heller, T. LAZAR-SHOEF et F. OMAR, Israeli Democracy Index 2017, Jerusalem, The Guttman Center for Public Opinion and Policy Research, 2017.

\section{- Rap avec David Grossman}

Le récit sur les artistes juifs cités jusqu'ici, qui appartiennent ou appartenaient à la culture politiquement dominante (l'engagement à droite ou l'indifférence), montre un glissement vers le désengagement politique, au sens du système représentatif traditionnel à l'échelle étatique - le même que celui observé au sein de la société israélienne en général ${ }^{9}$. Il existe bien entendu des voix alternatives qui se font entendre pour contester le 
consensus sur l'impuissance de la politique. Ces voix n'ont en revanche pas beaucoup d'espoir face à la politique telle qu'elle est pratiquée en Israël aujourd'hui, qu'elles jugent de plus en plus ouvertement raciste.

À la fin des années 1990, un groupe de personnes nées au milieu des années 1970 a commencé à jouer sous un nom de Hadag Nahash. Leur musique associait le Hip hop à des éléments de reggae, employait des instruments acoustiques, et les artistes mêmes portaient des tenues plutôt hippies - ce qui les rendait physiquement très différent'e-s des artistes associés à TACT, portant des vêtements typiques du rap américain (pantalons baggy, casquettes, et des bijoux - dans le cas de Subliminal, l'étoile de David).

Déjà assez connus après deux premiers albums, Hadag Nahash a enregistré en 2004 Shirat Hasticker (The Sticker Song), l'un des morceaux les plus marquants du rap israélien. Les paroles ont été écrites par le romancier David Grossman, et sont presque entièrement composées de citations des autocollants affichés sur les voitures israéliennes, une façon autrefois populaire d'exprimer ses opinions politiques, qu'elles soient de gauche ou de droite. La chanson est absurde telle quelle : les messages dont elle est tissée sont nécessairement contradictoires ("Toute la génération veut la paix ", "Pas de paix avec les Arabes "). Malgré cela, ou peut-être grâce à cela, elle permet de saisir le problème de la culture du slogan de la politique israélienne, de l'extrême simplification des revendications politiques dans la sphère publique. Elle est connue de pratiquement

\section{Les paroles de The Sticker Song ont été écrites par le romancier David Grossman, et sont presque entièrement composées de citations des autocollants affichés sur les voitures israéliennes.} tous les Israélien'ne·s, scandée par le public durant les concerts, et les contradictions qu'elle reflète semblent aujourd'hui paradoxalement admises comme telles, sans qu'il y ait de réelle volonté politique de s'y confronter. Elle ne prend pas parti (même si les artistes sont connus pour leurs positions de gauche), elle donne plutôt une image poétique de l'impossibilité du dialogue entre les Juif·ve·s israélien·ne·s eux·elles-mêmes.

\section{- Conclusion}

L'histoire du rap palestinien et israélien, le rap arabe et juif, est de ce fait une version condensée de l'histoire d'Israël depuis 1948. D'abord, une tentative de collaboration pacifique (1999-2000) brusquement terminée avec la deuxième Intifada qui a enflammé les passions politiques et a conduit à une série d'engagements soit en faveur de l'indépendance de la Palestine (l'Arabe T. Nafar, les Juifs A. Geffen et le groupe Hadag Nahash entre autres), soit allant dans le sens de la défense de la raison d'État israélienne (Subliminal et The Shadow). Ensuite, un retrait continu de l'engagement politique des Israélien.ne's jui.ve's de facto proches de 
10. A. Barell,

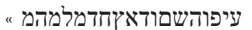

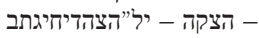
קראה ", Haaretz, 07/06/2018. la position politique dominante, ou le choix de ne pas en avoir du tout (Tuna, Nechi Nech). Un retrait accompagné par le rappel continu par les Palestinien'ne·s de leur présence, et par des voix discordantes juives qui continuent de se faire entendre.

La dépolitisation de la société israélienne peut se voir non seulement par le retour exclusif vers la vie familiale et les cercles d'ami-ess proches qui transparaît dans les choix musicaux mais aussi à travers la façon dont le grand public perçoit le fonctionnement des forces militaires, de l'armée israélienne de "défense ". Dans un texte récent, l'historien Ari Barrell note que l'efficacité de l'Iron Dome ${ }^{10}$, l'intercepteur de roquettes sophistiqué empêchant les missiles palestiniens de toucher le sol en les faisant exploser dans l'air, est à la fois une réalité et le symbole d'une situation politique de conflit profond, sans aucune progression possible. On peut ainsi caractériser le rap juif israélien très contemporain par un renoncement désespéré à la politique face à une situation insoluble et comme l'expression particulièrement poignante de l'insupportable blocage du conflit. 